\title{
Interplay of radiative and nonradiative transitions in surface hopping with radiation- molecule interactions
}

Juan José Bajo, Giovanni Granucci, and Maurizio Persico

Citation: The Journal of Chemical Physics 140, 044113 (2014); doi: 10.1063/1.4862738

View online: https://doi.org/10.1063/1.4862738

View Table of Contents: http://aip.scitation.org/toc/jcp/140/4

Published by the American Institute of Physics

\section{Articles you may be interested in}

Decoherence-induced surface hopping

The Journal of Chemical Physics 137, 22A545 (2012); 10.1063/1.4757100

Molecular dynamics with electronic transitions

The Journal of Chemical Physics 93, 1061 (1990); 10.1063/1.459170

Can we derive Tully's surface-hopping algorithm from the semiclassical quantum Liouville equation? Almost, but only with decoherence

The Journal of Chemical Physics 139, 214107 (2013); 10.1063/1.4829856

Perspective: Nonadiabatic dynamics theory

The Journal of Chemical Physics 137, 22 A301 (2012); 10.1063/1.4757762

Including quantum decoherence in surface hopping

The Journal of Chemical Physics 133, 134111 (2010); 10.1063/1.3489004

Direct semiclassical simulation of photochemical processes with semiempirical wave functions

The Journal of Chemical Physics 114, 10608 (2001); 10.1063/1.1376633

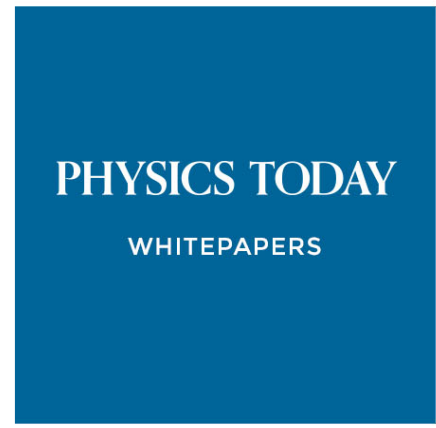

ADVANCED LIGHT CURE ADHESIVES

Take a closer look at what these environmentally friendly adhesive systems can do

\section{READ NOW}

PRESENTED BY (8) MASTERBOND 


\title{
Interplay of radiative and nonradiative transitions in surface hopping with radiation-molecule interactions
}

\author{
Juan José Bajo, ${ }^{1}$ Giovanni Granucci, ${ }^{2, a)}$ and Maurizio Persico ${ }^{2}$ \\ ${ }^{1}$ Departamento de Química-Física I, Universidad Complutense de Madrid, 28040 Madrid, Spain \\ ${ }^{2}$ Università di Pisa, Dipartimento di Chimica e Chimica Industriale, via Risorgimento 35, 56126 Pisa, Italy
}

(Received 20 November 2013; accepted 7 January 2014; published online 29 January 2014)

\begin{abstract}
We implemented a method for the treatment of field induced transitions in trajectory surface hopping simulations, in the framework of the local diabatization scheme, especially suited for on-the-fly dynamics. The method is applied to a simple one-dimensional model with an avoided crossing and compared with quantum wavepacket dynamics. The results show the importance of introducing a proper decoherence correction to surface hopping, in order to obtain meaningful results. Also the energy conservation policy of standard surface hopping must be revised: in fact, the quantum wavepacket energetics is well reproduced if energy absorption/emission is allowed for in the hops determined by radiation-molecule coupling. To our knowledge, this is the first time the issues of decoherence and energy conservation have been analyzed in depth to devise a mixed quantum-classical method for dynamics with molecule-field interactions. () 2014 AIP Publishing LLC. [http://dx.doi.org/10.1063/1.4862738]
\end{abstract}

\section{INTRODUCTION}

Mixed quantum-classical methods, employing classical nuclear trajectories propagated "on the fly," are nowadays routinely used for the theoretical study of the excited state dynamics of molecular systems. In most cases the light-induced excitation process does not need to be explicitly considered, and can be replaced by a sensible choice of starting conditions. However, sometimes the explicit inclusion of the interaction with radiation is needed or desirable: for example, in the simulation of quantum control or multiphoton processes. Several research groups have proposed different approaches to this problem, based on quantum-classical Liouville dynamics,,${ }^{1,2}$ mean field, ${ }^{3-5}$ or surface hopping (SH) ${ }^{6-19}$ In this contribution, we focus on the SH method, which is probably the most popular. SH was initially conceived for the description of nonradiative electronic transitions in molecular dynamics, and since then has benefited from 40 years of development. ${ }^{20-26}$ On the other hand, the explicit treatment of radiative (field induced) processes in $\mathrm{SH}$ is a relatively recent improvement, and several important issues need further consideration, especially when radiative and nonradiative transitions are taken into account concurrently.

Within the SH framework, we cite the approaches FISH (field induced surface hopping) $)^{7,16-19}$ and SHARC (SH in adiabatic representation including arbitrary couplings): ${ }^{12-15}$ beside being able to account for radiative and nonradiative transitions, both methods may also include spin-orbit couplings.

$\mathrm{SH}$ is a stochastic method in which a swarm of independent trajectories are run on the electronic potential energy surfaces (PESs) to simulate the dynamics of a molecular system. Nonadiabatic events are represented as "hops" between

${ }^{a)}$ Electronic mail: giovanni.granucci@unipi.it different PESs. In standard calculations, not taking into account the coupling with radiation, the conservation of the total energy of the system is enforced for each trajectory, even when a hop occurs. While this is far from being mandatory, it is however a simple way to achieve the basic requirement, namely, conservation of the average energy for the full swarm, still preserving the independency of the single trajectory. From this point of view, the case in which the interaction with the radiation field is explicitly included is more complicated, as both energy nonconserving (field-induced) and energy conserving (nonradiative) transitions are taken into account. The approach used by Richter et al. ${ }^{12}$ in this context was to enforce energy conservation after a hop only if the potential energy difference of the states involved lies outside the laser bandwidth: they were able to obtain a good agreement with full quantum results for the momentum probability distribution in the simulation of $\mathrm{IBr}$ photodissociation.

A known issue in $\mathrm{SH}$ is the lack of quantum decoherence, which originates from the fact that, for a given trajectory, the probability amplitudes for all the electronic states refer to the same nuclear phase space point and are fully coupled. The methods used to amend this problem are usually based on energetic criteria, enforcing decoherence by collapse of the electronic wavefunction on the "current" state (the one on which PES the trajectory is running), when the electronic energy differences are large. ${ }^{27-29}$ Other recipes require the conservation of the total energy along a trajectory, and therefore cannot be applied without modifications in the present context. ${ }^{30-32}$ In general, consideration of decoherence in SH appears to be very important when field-induced transitions are involved: in fact, the nonadiabatic events following the initial excitation are poorly described if one does not take into account that the ground state wavepacket usually propagates very differently from the excited states ones. An important exception may be offered by simulations aimed at reproducing the 
control of molecular processes by shaped laser fields, as in that case one is usually interested in maximizing the population of a given excited state, while the ground state population can be disregarded in the subsequent nonadiabatic dynamics. Nevertheless, as recognized by Tavernelli and co-workers ${ }^{10}$ in performing local control theory calculations on $\mathrm{LiF}$, even in that case decoherence effects may have some minor impact.

In the present contribution, we introduce the interaction with the radiation field in the framework of our local diabatization (LD) on-the-fly scheme for $\mathrm{SH}^{33,34}$ The method is applied to a one-dimensional model system, which allows to address the problems outlined above of energy (non)conservation and lack of quantum decoherence, and to compare the $\mathrm{SH}$ results with quantum dynamics calculations.

\section{METHOD}

In this section, we briefly recall Tully's "fewest switches" version of the $\mathrm{SH}$ method ${ }^{21}$ and the LD algorithm, ${ }^{33}$ focusing on the modifications we have introduced to account for the interaction with an external electric field.

Let $\hat{\mathcal{H}}_{e l}$ be the electronic Hamiltonian of the molecular system considered, in the absence of the field, and $\varphi_{K}$ $(K=1 \ldots N)$ a set of $N$ (approximate) eigenstates of $\hat{\mathcal{H}}_{e l}$, with eigenvalues $U_{K}$, spanning the electronic subspace $\mathcal{S}(t)$ of interest. Note that $\hat{\mathcal{H}}_{e l}$ may include the spin-orbit interaction as well. ${ }^{35}$ The total Hamiltonian is then $\hat{\mathcal{H}}=\hat{\mathcal{H}}_{e l}+\hat{\mathcal{H}}_{\text {ext }}$, where

$$
\hat{\mathcal{H}}_{\text {ext }}=-\vec{E}(t) \cdot \vec{\mu}
$$

describes the interaction with the external electric field $\vec{E}(t)$, $\vec{\mu}$ being the molecular dipole operator. In the SH scheme, the nuclear motion is subject to the classical Newton equations. In particular, a given nuclear trajectory $\mathbf{Q}(t)$ is evolved on a single adiabatic PES $U_{K}(\mathbf{Q})$, but it may hop to another surface at any time. The electronic motion is described by a wavefunction expanded in terms of the adiabatic basis considered

$$
\Psi(t)=\sum_{K}^{N} A_{K}(t) \varphi_{K}(\mathbf{Q}(t)) .
$$

The complex coefficients $A_{K}(t)$ are obtained inserting $\Psi(t)$ in the time dependent Schrödinger equation (TDSE) for the electrons only ${ }^{21}$

$$
\dot{A}_{K}=-\frac{i}{\hbar} A_{K} U_{K}-\sum_{L} A_{L}\left(G_{K L}+\frac{i}{\hbar} H_{K L}^{e x t}\right),
$$

where $G_{K L}$ and $H_{K L}^{e x t}$ are

$$
G_{K L}=\left\langle\varphi_{K} \mid \frac{\partial \varphi_{L}}{\partial t}\right\rangle, \quad H_{K L}^{e x t}=-\vec{E}(t) \cdot\left\langle\varphi_{K}|\vec{\mu}| \varphi_{L}\right\rangle .
$$

In the LD scheme, the integration of Eq. (3) is performed in an alternative "locally diabatic" $\mathrm{N}$-dimensional electronic basis $|\boldsymbol{\eta}\rangle$, spanning the same subspace $\mathcal{S}$ as $|\boldsymbol{\varphi}\rangle$, and defined so as to be approximately constant in the integration time step $\Delta t$ (hereafter we set $t=0$ at the beginning of the time step for simplicity):

$$
\begin{aligned}
|\boldsymbol{\eta}(0)\rangle & =|\varphi(0)\rangle \\
|\boldsymbol{\eta}(\Delta t)\rangle & \equiv|\boldsymbol{\eta}(0)\rangle=|\varphi(\Delta t)\rangle \mathbf{T}^{+}(\Delta t),
\end{aligned}
$$

where the unitary matrix $\mathbf{T}(\Delta t)$ is obtained by Löwdin orthonormalization of the overlap $\mathbf{S}=\langle\varphi(0) \mid \varphi(\Delta t)\rangle$. At the beginning of the time step we have, by definition, $\mathbf{T}(0)=\mathbf{1}$. In the LD basis, the integration of the electronic TDSE is particularly easy. In fact, the dynamic couplings $\left\langle\eta_{I} \mid \partial \eta_{L} / \partial t\right\rangle$ vanish by construction, while the analogous couplings $G_{K L}$ in the adiabatic basis give rise to numerical problems in regions close to PES crossings. In particular, by expanding $\Psi(t)$ in the locally diabatic basis

$$
\Psi(t)=\sum_{K}^{N} D_{K}(t) \eta_{K}
$$

we get

$$
\dot{\mathbf{D}}=-\frac{i}{\hbar} \mathbf{H D}, \quad \mathbf{H}=\mathbf{H}_{0}-\vec{E} \cdot \overrightarrow{\mathbf{M}}
$$

where the matrices $\mathbf{H}_{0}$ and $\overrightarrow{\mathbf{M}}$ are defined at the beginning and at the end of the integration time step

$$
\begin{gathered}
\mathbf{H}_{0}(0)=\mathbf{U}(0) \quad \overrightarrow{\mathbf{M}}(0)=\langle\varphi(0)|\vec{\mu}| \varphi(0)\rangle, \\
\mathbf{H}_{0}(\Delta t)=\mathbf{T}(\Delta t) \mathbf{U}(\Delta t) \mathbf{T}^{+}(\Delta t), \\
\overrightarrow{\mathbf{M}}(\Delta t)=\mathbf{T}(\Delta t)\langle\boldsymbol{\varphi}(\Delta t)|\vec{\mu}| \varphi(\Delta t)\rangle \mathbf{T}^{+}(\Delta t) .
\end{gathered}
$$

In the above equations $\mathbf{U}$ is the diagonal matrix collecting the adiabatic energies $U_{K}$. Taking advantage of the invariance of the LD basis, $\mathbf{H}$ can be obtained at intermediate times by linear interpolation

$$
\begin{aligned}
\mathbf{H}(t) \simeq \mathbf{H}(0)+\left[\mathbf{H}_{0}(\Delta t)-\mathbf{H}_{0}(0)\right] \frac{t}{\Delta t} \\
-\vec{E}(t) \cdot[\overrightarrow{\mathbf{M}}(\Delta t)-\overrightarrow{\mathbf{M}}(0)] \frac{t}{\Delta t},
\end{aligned}
$$

where of course $\mathbf{H}(0)=\mathbf{H}_{0}(0)-\vec{E}(0) \cdot \overrightarrow{\mathbf{M}}(0)$. Equation (8) can be integrated by a simple approximate formula:

$$
\mathbf{D}(\Delta t) \simeq \exp \left(-\frac{i}{\hbar} \mathbf{H}(\Delta t / 2) \Delta t\right) \mathbf{D}(0) .
$$

From the diabatic coefficients one then gets the adiabatic ones: $\mathbf{A}(\Delta t)=\mathbf{T}^{+}(\Delta t) \mathbf{D}(\Delta t)$. Equation (13) yields accurate results when $\mathbf{H}$ changes slowly with time in the interval $\Delta t$; this is indeed the case for the locally diabatic energies and dipoles, but not for the electric field $\vec{E}(t)$, which may oscillate with a period of the same order of magnitude of $\Delta t .^{36} \mathrm{We}$ resort, therefore, to the following ansatz

$$
\begin{aligned}
\mathbf{D}(\Delta t) \simeq & \exp \left(-\frac{i}{\hbar} \mathbf{H}\left(t_{n_{s}}\right) \Delta t^{\prime}\right) \ldots \exp \left(-\frac{i}{\hbar} \mathbf{H}\left(t_{j}\right) \Delta t^{\prime}\right) \ldots \\
& \times \exp \left(-\frac{i}{\hbar} \mathbf{H}\left(t_{1}\right) \Delta t^{\prime}\right) \mathbf{D}(0), \\
\Delta t^{\prime}= & \frac{\Delta t}{n_{s}}, \quad t_{j}=\frac{2 j-1}{2 n_{s}} \Delta t \quad\left(j=1 \ldots n_{s}\right),
\end{aligned}
$$

which amounts to integrating the electronic TDSE with a smaller time step $\Delta t^{\prime}$. Note, however, that the relevant electronic quantities (energies, wavefunctions, and couplings) need to be evaluated only at $\Delta t$ time intervals (thanks to 
the LD scheme), and the number of electronic states considered is usually small: as a consequence, the parameter $n_{s}$ can be chosen large enough as to perform a very accurate integration of the TDSE within the approximation (12), without noticeably increasing the computational cost of a dynamic simulation performed on-the-fly.

Following Tully's fewest switches prescription, ${ }^{21,24,37}$ the transition probability $P_{K \rightarrow L}$ from the current electronic state $K$ to state $L$ is given by

$$
P_{K \rightarrow L}=\frac{\max \left\{0, \int_{0}^{\Delta t} B_{K L} d t\right\}}{p_{K}(0)},
$$

where $p_{K}(0)=\left|A_{K}(0)\right|^{2}$ is the population of state $K$ and the integral accounts for the variation of $B_{K L}$ (the rate of decrement of $p_{K}$, due to state $L$ ) within a time step. We have

$$
\begin{gathered}
\dot{p}_{K}=-\sum_{L} B_{K L}, \quad B_{K L}=-B_{L K}, \\
B_{K L}=2 \Re\left\{\rho_{L K} G_{K L}\right\}-\frac{2}{\hbar} \Im\left\{\rho_{L K} H_{K L}^{e x t}\right\} .
\end{gathered}
$$

In the above equation, we made use of the electronic density matrix $\rho_{K L}=A_{K} A_{L}^{*}$. The integral appearing in Eq. (16) can be computed numerically, exploiting the same partition of the time interval $\Delta t$ in $n_{s}$ substeps $\Delta t^{\prime}$ introduced above for the integration of the TDSE (see Eq. (15)). The diagonalization of $\mathbf{H}_{0}\left(t_{j}\right)$ yields $\mathbf{T}\left(t_{j}\right)$ which is in turn exploited to evaluate the adiabatic quantities $\rho_{L K}\left(t_{j}\right), H_{K L}^{\text {ext }}\left(t_{j}\right)$, and $G_{K L}\left(t_{j}\right)$, needed in Eq. (18) for $B_{K L}\left(t_{j}\right)$. Note in particular that the $G_{K L}$ couplings, not computed in the LD algorithm for the TDSE integration, can be obtained considering that, as far as the LD basis is invariant in the time step $\Delta t$

$$
\mathbf{G}=-\dot{\mathbf{T}}^{+} \mathbf{T} .
$$

Therefore, a numerical approximation for $\mathbf{G}\left(t_{j}\right)$ which keeps the antihermiticity (needed for the antisymmetry condition (17) to be satisfied) is

$$
\mathbf{G}\left(t_{j}\right)=\frac{\mathbf{T}^{+}\left(t_{j-1}\right) \mathbf{T}\left(t_{j}\right)-\mathbf{T}^{+}\left(t_{j}\right) \mathbf{T}\left(t_{j-1}\right)}{2 \Delta t^{\prime}} .
$$

For notational simplicity we define $\bar{B}_{K L}=\int_{0}^{\Delta t}$ $B_{K L} d t / \Delta t$. According to Eq. (18), $\bar{B}_{K L}$ is partitioned in two contributions: the first one, $\bar{B}_{K L}^{\text {mol }}$, is due to the nonadiabatic couplings and the second one, $\bar{B}_{K L}^{\text {ext }}$, to the external electric field. This allows for a distinction between radiative (field induced) and nonradiative electronic transitions. In particular, the surface hop from state $K$ to state $L$ is labeled as radiative or nonradiative if $\bar{B}_{K L}^{e x t}>\bar{B}_{K L}^{\text {mol }}$ or $\bar{B}_{K L}^{m o l}>\bar{B}_{K L}^{e x t}$, respectively. For nonradiative hops, we impose the conservation of the total energy, i.e., the module of the nuclear velocity is rescaled in order to compensate for the sudden variation of the electronic energy (and, as usual in SH, a nonradiative upward hop is forbidden if the nuclear kinetic energy is not sufficient for such a compensation). The radiative hops, on the contrary, do not entail any action to enforce energy conservation and are never forbidden.

In Ref. 33, the transition probability $P_{K \rightarrow L}$ was obtained in a different way, i.e., by directly looking at the change of the state $K$ population in a time step, expressing it in terms of the unitary matrix connecting the coefficients $\mathbf{A}(0)$ and $\mathbf{A}(\Delta t)$, and proposing a partition of that expression in contributions pertaining to the electronic states $L \neq K$. The present algorithm represents an improvement, in that it allows to distinguish between radiative and nonradiative contributions. Moreover, expressing the change of current state probability without explicit reference to the LD basis (see Eqs. (17) and (18)) allows to partition it in a physically sound way, ${ }^{21}$ avoiding the appearance of unwanted "diagonal" $(K \rightarrow K)$ terms.

Our overlap driven decoherence correction (ODC) scheme $^{32}$ is easily adapted for taking into account the interaction with the laser field. Let $K$ be the current state, and assume the $j$ th ancillary frozen Gaussian wavepacket

$$
G_{L, j}(x)=\left(\frac{2 a}{\pi}\right)^{1 / 4} \exp \left\{-a\left(x-Q_{L, j}\right)^{2}+i x P_{L, j} / \hbar\right\}
$$

is just spawned on state $L$. According to the ODC algorithm, initially $Q_{L, j}$ is set equal to the current nuclear position $Q$ for state $K$ (for simplicity, we consider here only one dimension, the extension to the multidimensional case being straightforward). In the absence of the laser field, the momentum $P_{L, j}$ is obtained from the current nuclear momentum $M \dot{Q}$ rescaled to allow for energy conservation; in the present context the rescaling is only done when $\bar{B}_{K L}^{m o l}>\bar{B}_{K L}^{e x t}$. Moreover, the Gaussian wavepacket parameters $P_{L, j}$ and $Q_{L, j}$ are not propagated in time using the "zeroth order" approximation of Ref. 32, since this was based on the momentum change required by energy conservation. Rather, we resort to Heller's evolution for frozen Gaussian wavepackets ${ }^{38,39}$

$$
\begin{aligned}
\dot{P}_{L, j} & =-\frac{d U_{L}(Q)}{d Q}, \\
\dot{Q}_{L, j} & =P_{L, j} / M .
\end{aligned}
$$

To avoid increasing the computational burden, the gradients $d U_{L} / d Q$ are approximated as those of the current nuclear position $Q$ for state $K$, instead of computing them at $Q_{L, i}$. This is a good approximation as far as the $Q_{L, i}$ are close to the current nuclear position. When they diverge, the ancillary wavepackets are anyway discarded. The Newton equations (22) and (23) are integrated using the standard velocity Verlet algorithm. ${ }^{40}$ In on-the-fly dynamics, this procedure is anyway more computationally demanding than the zeroth order approximation referred above, as it requires, in principle, the evaluation of the gradients of all the other PESs, in addition to that of the current state. While this approach is still viable in a semiempirical framework, as the one considered in Sec. III, it may become too expensive in an ab initio context.

\section{TWO STATE, ONE-DIMENSIONAL MODEL SYSTEM}

We applied the method described in Sec. II to a onedimensional model system, which allowed us to compare the $\mathrm{SH}$ results to the full quantum time evolution (conducted with the same $\hat{\mathcal{H}}_{\text {ext }}$ of Eq. (1)). The model system corresponds to the one we already used in Ref. 32, and it is appropriate for the 


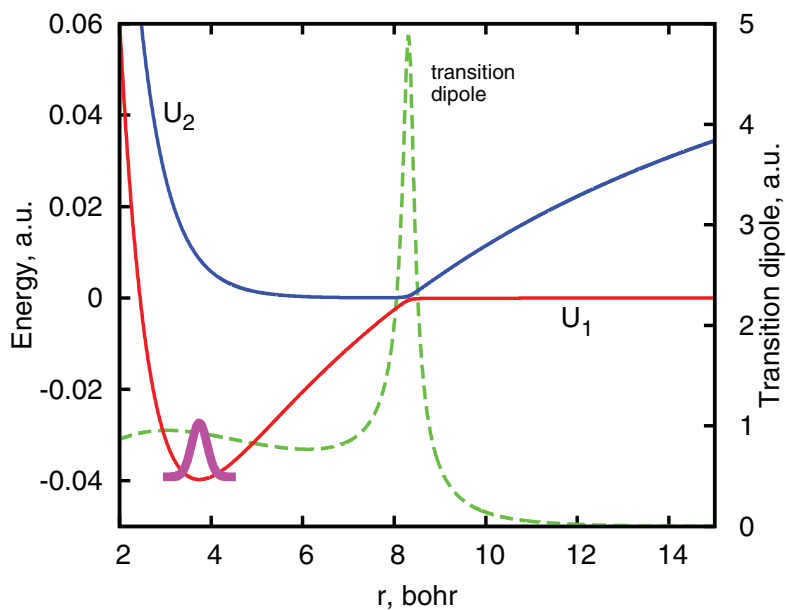

FIG. 1. Model system considered in this study. Presented are the adiabatic potential energy curves (solid lines) and the transition dipole $\mu_{12}$ (dashed line). $U_{1}$ and $U_{2}$ label the lower and the upper state PES, respectively. The squared module of the starting wavepacket is also shown (thick line).

description of ionic/covalent crossing in alkali halides. The PES and couplings were obtained on the fly using our Floating Occupation Molecular Orbitals Configuration Interaction (FOMO-CI) and an AM1 semiempirical Hamiltonian, with semiempirical parameters modified by us to yield potential energy curves roughly similar to those of LiF. The reduced mass of the diatomic has been set to $m=14583$ a.u. ( $8 \mathrm{amu}$ ). In this way, the lower state minimum at $r_{0}=3.743$ bohrs is characterized by an harmonic frequency $\omega_{0}=262 \mathrm{~cm}^{-1}$. The starting wavepacket lies on the lower state PES and corresponds to the ground state of a harmonic oscillator of mass $m$ and frequency $\omega_{0}$, centered in $r_{0}$. We show in Figure 1 the energies of the two electronic states considered, together with the starting wavepacket and the transition dipole moment $\mu_{12}=\left\langle\varphi_{1}\left|\mu_{\|}\right| \varphi_{2}\right\rangle$ (note that, as both states have $\Sigma$ symmetry, the transition dipole moment is parallel to the internuclear axis).

The electric field $\vec{E}(t)$ is linearly polarized along the internuclear axis of the molecule, the orientation of which is assumed fixed along the $x$ axis. In particular we set

$$
E_{x}(t)=E_{0} e^{-2 \ln (2)\left(\frac{t-t_{0}}{\tau_{p}}\right)^{2}} \cos (\Omega t), \quad E_{y}=E_{z}=0,
$$

where $E_{0}$ is the maximum amplitude of the electric field, $\tau_{p}$ is the pulse length (full width at half maximum of the squared Gaussian envelope), and $\Omega$ is the carrier wave frequency, set so as to be in resonance with the $1 \rightarrow 2$ transition at $r_{0}: \hbar \Omega$ $=U_{2}\left(r_{0}\right)-U_{1}\left(r_{0}\right)=0.04824$ a.u. Four sets of simulations have been performed, each one characterized by different values of the pulse parameters. In particular, for the amplitude $E_{0}$ we used 0.0015 and 0.015 a.u. (corresponding, respectively, to peak powers $I_{p}$ of about 0.079 and $7.9 \mathrm{TW} / \mathrm{cm}^{2}$ ). For the pulse length $\tau_{p}$ we used 500 and 6000 a.u., with $t_{0}=2000$ and 10000 a.u., respectively.

The full quantum calculations have been carried out by expressing the starting wavepacket in the diabatic basis ${ }^{32}$ and performing the time evolution on a grid with a second order split-operator algorithm. ${ }^{41}$ The results are then presented by reverting back the quantum wavefunction $\Psi_{q}$ to the adiabatic basis:

$$
\Psi_{q}(r, t)=\chi_{1}(r, t)\left|\varphi_{1}\right\rangle+\chi_{2}(r, t)\left|\varphi_{2}\right\rangle .
$$

For the SH simulations we used swarms of 10000 trajectories; the initial conditions were obtained by sampling the Wigner distribution corresponding to the starting quantum wavepacket. ${ }^{42}$ The integration time step was $\Delta t=0.1 \mathrm{fs}$, and we set $n_{s}=20$ (see Sec. II). After some test calculations, the parameters representing the overlap threshold $\left(S_{\min }\right)$ and the width $(\sigma)$ of the ancillary Gaussian wavepackets used in the ODC scheme ${ }^{32}$ were set to $10^{-8}$ and 0.5 a.u., respectively.

In Figure 2, we show the population of the upper state as a function of time, for the four simulations considered. In the full quantum case, $p_{2}^{q}(t)=\int\left|\chi_{2}(r, t)\right|^{2} d r$ is shown. For SH, we report the fraction of trajectories $\Pi_{2}$ running on $U_{2}$ and the upper state population averaged over the swarm of trajectories $\left\langle p_{2}\right\rangle$ (the latter quantity is omitted for clarity when the ODC decoherence correction is used, as it is almost coincident with $\Pi_{2}$ ).

Let us consider first the case in which the laser pulse has the weakest amplitude and shortest FWHM (top left panel of Figure 2). Approximately, the laser field is only present in the time range $25-70 \mathrm{fs}$; it is able to transfer about $18 \%$ of the population to the upper state. At times $t>70 \mathrm{fs}$ we are, therefore, left with an almost stationary wavepacket occupying the minimum energy region of the ground state, representing $82 \%$ of the total population and with vanishingly small coupling to the upper state wavepacket. It is, therefore, the latter which, reaching the avoided crossing region at $r_{c}=8.32$ bohrs, gives rise to the nonradiative population transfer at $t=150-200 \mathrm{fs}$ and reduces $p_{2}^{q}$ to 0.026 . The wavepacket just created on the lower state then proceeds to dissociation. Note that the Landau Zener adiabatic transition probability (starting from the Franck-Condon point with zero kinetic energy) is $p_{L Z}=0.855$, which is in quantitative agreement with the drop of $p_{2}^{q}$ from 0.18 to 0.026 . The wavepacket remained in the upper state cannot dissociate: therefore, it goes back to the avoided crossing region, giving rise to the population transfer at $t=260-300 \mathrm{fs}$, and so on. While the nonadiabatic quantum wavepacket dynamics is very well reproduced by the SH method with ODC, without decoherence correction SH fails badly because the trajectories go through the avoided crossing with substantial amplitudes in both electronic states. For example, the trajectories initially excited to the upper state reach the avoided crossing region with $p_{1}=0.80$ and $p_{2}=0.20$ in the average, so they behave more as if they belonged to the ground state than to the excited one: by crossing the strong interaction region, $\left\langle p_{2}\right\rangle$ further increases instead of decreasing, and too few downward hops take place.

The decoherence problems outlined above are alleviated if more population is transferred to the upper state during the short pulse: this is actually what happens for $E_{0}=0.015$ a.u. (middle left panel of Figure 2). Anyway, ODC improves considerably the SH results even in this case, quantitatively accounting for radiative and non radiative transfer of population. Would the pulse be optimized (as to intensity, tuning, duration, etc.) to transfer all the population to the upper state, as in a coherent control experiment, the decoherence correction 

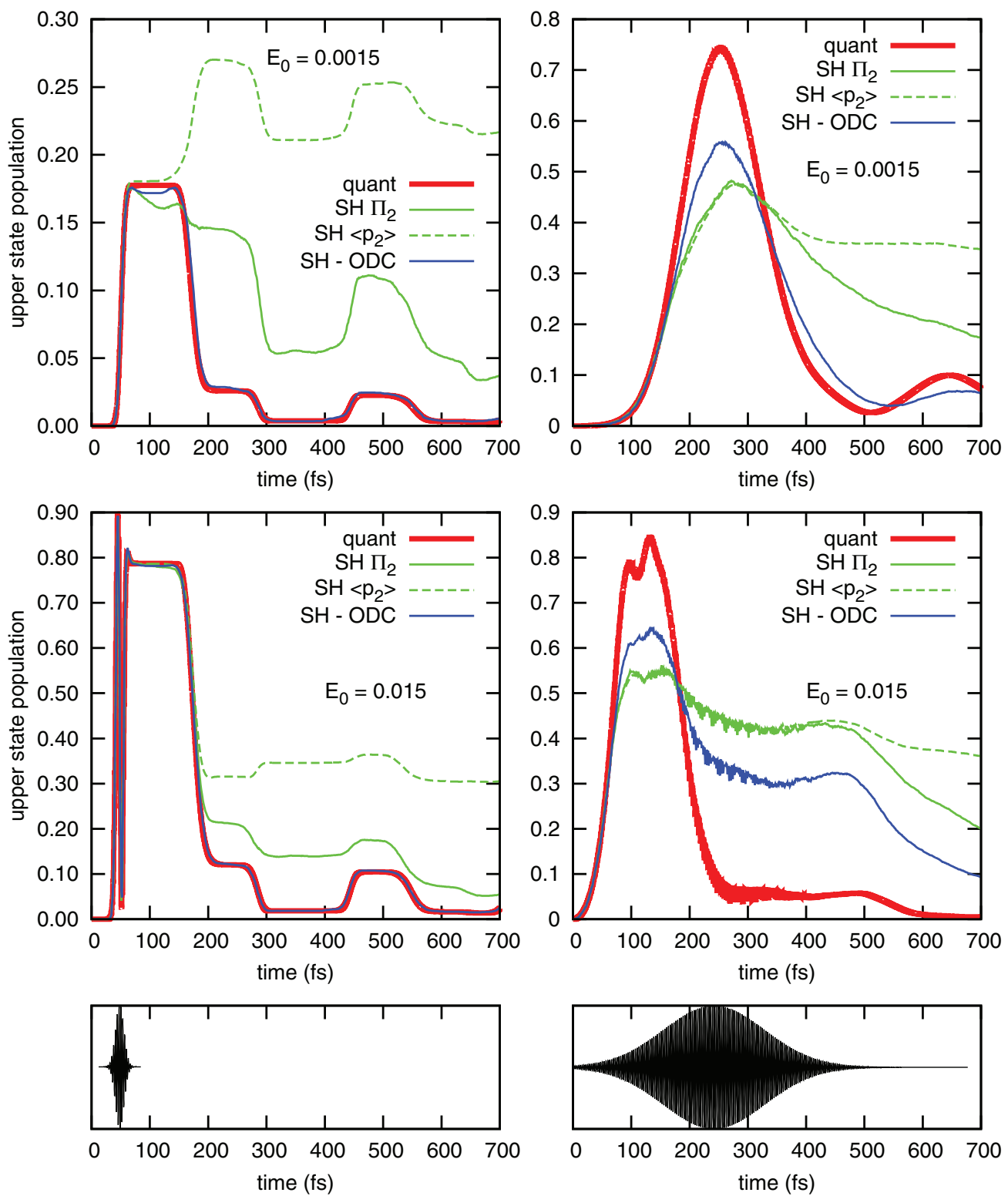

FIG. 2. Upper state population versus time. Top (middle) panels: results for $E_{0}=0.0015$ (respectively, $E_{0}=0.015$ a.u.). Bottom panels: pulse shapes. Red thick lines: full quantum results. Blue solid lines: SH with ODC decoherence correction, fraction of trajectories on the upper state $\left(\Pi_{2}\right)$. Green solid $($ dashed) lines: $\mathrm{SH}$ without decoherence correction, $\Pi_{2}$ (respectively, $\left\langle p_{2}\right\rangle$, see text).

should not be needed anymore, at least up to the first crossing of the strong interaction region.

With longer pulses $\left(\tau_{p}=6000\right.$ a.u., right column of Figure 2) the agreement deteriorates. At least in part, this is related to the effect of the displacement of the wavepacket in the excited state during the laser pulse, which is not faithfully reproduced by the trajectory swarm. Going back to the test with $\tau_{p}=500$ a.u. and $E_{0}=0.015$, we clearly see that the upper state probability undergoes Rabi-like oscillations. In a two-level system (no nuclear dynamics), with the transition dipole $\mu\left(r_{0}\right)=0.928$ a.u., this would be a $3.34 \pi$ pulse, i.e., it would produce a final probability $p_{2}=\sin ^{2}(3.34 \pi)$ $=0.75$, after 3 complete population switches. With a repulsive potential in the upper state, such Rabi-like oscillations obey the simple two-state rule only if the pulse is very short, ${ }^{43,44}$ otherwise the wavepacket starts moving out of the FranckCondon region, i.e., out of resonance, during the time the field is on. This effect is much more important for the 6000 a.u. pulses and is underestimated by the semiclassical treatment. In our tests, the carrier wave is in resonance with the $1 \rightarrow 2$ transition at $r_{0}$; as a consequence, the lowest energy trajectories, oscillating in the close proximity of $r_{0}$, are preferably excited (note that the Wigner sampling of the $v=0$ eigenstate of the harmonic oscillator yields a distribution of energies $\left.P(E) \propto e^{-2 E / \hbar \omega}\right)$. Let us now focus on the first $150 \mathrm{fs}$ of the test with $E_{0}=0.0015$ a.u. (top right panel of Figure 2). During this time the nonadiabatic effects are negligible, because the quantum or classical wavepackets have not reached the avoided crossing region. Considering the trajectories that are excited before $t=150 \mathrm{fs}$, the averaged kinetic energy at the time of the first upward hop is $0.266 \mathrm{mEh}$, noticeably less than in the initial swarm in the ground state $(0.307 \mathrm{mEh})$. So, the SH trajectories promoted to the upper state at the beginning of the dynamics are characterized by an average 
kinetic energy $\left\langle K_{2}\right\rangle$ lower than that of the corresponding quantum wavepacket: for instance, at $t=150 \mathrm{fs},\left\langle K_{2}\right\rangle$ $=3.62 \mathrm{mEh}$ while $\left\langle\chi_{2}\left|\hat{p}^{2} / 2 m\right| \chi_{2}\right\rangle /\left\langle\chi_{2} \mid \chi_{2}\right\rangle=4.68 \mathrm{mEh}$. The upper state trajectory swarm is then slower than the quantum wavepacket, and spends more time in the quasi-resonance region. Its Rabi-like oscillations are less disturbed by the nuclear motion and the final population of the excited state is different from the quantum one. Clearly, as $\tau_{p}$ increases, the resonance condition is imposed more sharply, while the excited molecule has more time to elongate the bond and get out of resonance, so this effect becomes more important.

Even with the differences outlined above, the $\mathrm{SH}$ trajectories reproduce fairly well the behavior of the quantum population with $\tau_{p}=6000$ a.u. and the weaker field intensity (top right panel of Figure 2). The decoherence correction still improves very much the $\mathrm{SH}$ results. With the stronger field $\left(E_{0}=0.015\right.$, middle right panel of Figure 2$)$ we obtain a poorer agreement. In that case, due to the large intensity of the radiation, it is harder to follow in detail the wavepacket dynamics; probably interference effects between wavepacket components created by radiative and non-radiative transitions play a non negligible role.

In Table I, we report the dissociation probability $p_{\text {diss }}$ together with the average kinetic energy $K_{\text {diss }}$ of the fragments, which would represent an experimentally measurable outcome for a system of this kind. The dissociation threshold is arbitrarily set to $r=12$ bohrs, and the results of Table I are obtained at $t=700 \mathrm{fs}=28940$ a.u. In the quantum wavepacket treatment, $p_{\text {diss }}=\left\langle\chi_{1 \text {, diss }} \mid \chi_{1 \text {, diss }}\right\rangle$, where $\chi_{1}$, diss is the portion of $\chi_{1}$ at $r>12$ bohrs (we remind that in the present simulations dissociation can only be obtained on the ground state PES), and $K_{\text {diss }}$ $=\left\langle\chi_{1, \text { diss }}\left|\hat{p}^{2} / 2 m\right| \chi_{1, \text { diss }}\right\rangle /\left\langle\chi_{1, \text { diss }} \mid \chi_{1, \text { diss }}\right\rangle$. Similarly, in the $\mathrm{SH}$ treatments, $p_{\text {diss }}$ is the fraction of trajectories with $r>12$ bohrs, and $K_{\text {diss }}$ their averaged kinetic energy.

Considering $p_{\text {diss }}$, as already discussed above (see Figure 2), the SH results reproduce quantitatively the quantum ones for the shortest pulse, and the agreement deteriorates for the longest pulse, especially with high intensity. The kinetic energy of the dissociated fragment $K_{\text {diss }}$ shows a more uniform agreement: the largest relative difference between quantum and $\mathrm{SH}$ results amounting to $9 \%$. We also performed $\mathrm{SH}$ calculations suppressing the conservation of the total energy in nonradiative hops (labeled as $\mathrm{SH}^{\text {nec }}$ in Table I). While this has almost no effect on the dissociation probability, it is important for the kinetic energy of the fragment: the $\mathrm{SH}^{\text {nec }}$

TABLE I. Dissociation probabilities $\left(p_{\text {diss }}\right)$ and kinetic energies of the fragments $\left(K_{\text {diss }}, \mathrm{mEh}\right)$ at $t=700 \mathrm{fs}$. $\mathrm{SH}^{\text {nec }}$ labels $\mathrm{SH}$ results obtained without imposing energy conservation in non radiative transitions.

\begin{tabular}{lrlcccccc}
\hline \hline & & \multicolumn{3}{c}{$p_{\text {diss }}$} & & \multicolumn{3}{c}{$K_{\text {diss }}$} \\
\cline { 3 - 5 } \cline { 7 - 9 }$E_{0}$ & \multicolumn{1}{c}{$\tau_{p}$} & Quant & $\mathrm{SH}$ & $\mathrm{SH}^{\text {nec }}$ & & Quant & $\mathrm{SH}$ & $\mathrm{SH}^{\text {nec }}$ \\
\hline 0.0015 & 500 & 0.158 & 0.155 & 0.152 & & 8.801 & 8.938 & 7.765 \\
0.0015 & 6000 & 0.848 & 0.647 & 0.635 & & 9.173 & 8.652 & 7.705 \\
0.015 & 500 & 0.698 & 0.694 & 0.693 & & 8.027 & 8.138 & 6.945 \\
0.015 & 6000 & 0.865 & 0.513 & 0.503 & & 8.429 & 7.634 & 7.122 \\
\hline \hline
\end{tabular}

results for $K_{\text {diss }}$ differ from the quantum values much more than the SH ones. This shows that, in the simulation of energy disposal with surface hopping, it is important to distinguish between radiative and nonradiative transitions, and to enforce energy conservation in the latter.

\section{CONCLUSIONS}

In this work, we described a method for nonadiabatic molecular dynamics simulations with explicit inclusion of radiation induced transitions, in the framework of SH. The implementation has been performed according to our local diabatization scheme, most useful in on-the-fly calculations as it allows to use large integration time steps. ${ }^{33,34}$ The method has been applied to a one-dimension two-state model system including an avoided crossing, in which both radiative (field induced) and nonradiative transitions are important. Even within this simple model, we were able to point out some issues, to our knowledge not yet addressed in the literature, concerning the ability of surface hopping with field-molecule interactions to reproduce quantum wavepacket dynamics.

First, any partial switch of population caused by radiative excitation, followed by nonadiabatic dynamics, entails effects of quantum decoherence between the amplitudes in the two electronic states that are not properly dealt with by standard surface hopping. Using our ODC, ${ }^{32}$ suitably adapted to the present context, we were able to accurately reproduce the quantum results at least for very short pulses. With longer pulses, the agreement was less good, but the ODC anyway improved it.

Another issue concerns energy conserving (nonradiative) and energy non conserving (radiative) transitions: this distinction has to be taken into account in $\mathrm{SH}$ in order to correctly reproduce the energetics of the quantum wavepackets, and we propose a simple recipe to this aim. Finally, minor discrepancies arise from how the interplay of optical excitation and nuclear motion is treated in SH and in quantum wavepacket dynamics.

\section{ACKNOWLEDGMENTS}

J.J.B. acknowledges a grant from the Universidad Complutense of Madrid, G.G. and M.P. acknowledge grants from the University of Pisa.

${ }^{1}$ N. Rekik, C.-Y. Hsieh, H. Freedman, and G. Hanna, J. Chem. Phys. 138, 144106 (2013).

${ }^{2}$ F. Martinez, N. Rekik, and G. Hanna, Chem. Phys. Lett. 573, 77 (2013).

${ }^{3}$ T. Yonehara, K. Hanasaki, and K. Takatsuka, Chem. Rev. 112, 499 (2012).

${ }^{4}$ K. Yagi and K. Takatsuka, J. Chem. Phys. 123, 224103 (2005).

${ }^{5}$ T. Yonehara and K. Takatsuka, J. Chem. Phys. 128, 154104 (2008).

${ }^{6}$ G. A. Jones, A. Acocella, and F. Zerbetto, J. Phys. Chem. A 112, 9650 (2008).

${ }^{7}$ R. Mitrić, J. Petersen, and V. Bonačić-Koutecký, Phys. Rev. A 79, 053416 (2009).

${ }^{8}$ J. Petersen, R. Mitrić, V. Bonačić-Koutecký, J.-P. Wolf, J. Roslund, and H. Rabitz, Phys. Rev. Lett. 105, 073003 (2010).

${ }^{9}$ I. Tavernelli, B. F. E. Curchod, and U. Rothlisberger, Phys. Rev. A 81, 052508 (2010).

${ }^{10}$ B. F. E. Curchod, T. J. Penfold, I. Tavernelli, and U. Rothlisberger, Phys. Rev. A 84, 042507 (2011).

${ }^{11}$ R. Mitrić, J. Petersen, M. Wohlgemuth, U. Werner, V. Bonačić-Koutecký, L. Wöste, and J. Jortner, J. Phys. Chem. A 115, 3755 (2011). 
${ }^{12}$ M. Richter, P. Marquetand, J. González-Vázquez, I. R. Sola, and L. González, J. Chem. Theory Comput. 7, 1253 (2011).

${ }^{13}$ P. Marquetand, M. Richter, J. González-Vázquez, I. R. Sola, and L. González, Faraday Discuss. 153, 261 (2011).

${ }^{14}$ J. J. Bajo, J. González-Vázquez, I. R. Sola, J. Santamaria, M. Richter, P. Marquetand, and L. González, J. Phys. Chem. A 116, 2800 (2012).

${ }^{15}$ D. Geißler, P. Marquetand, J. González-Vázquez, L. González, T. Rozgonyi, and T. Weinacht, J. Phys. Chem. A 116, 11434 (2012).

${ }^{16}$ G. Tomasello, M. Wohlgemuth, J. Petersen, and R. Mitrić, J. Phys. Chem. B 116, 8762 (2012).

${ }^{17}$ J. Petersen and R. Mitrić, Phys. Chem. Chem. Phys. 14, 8299 (2012).

${ }^{18}$ J. Petersen, M. Wohlgemuth, B. Sellner, V. Bonačić-Koutecký, H. Lischka, and R. Mitrić, Phys. Chem. Chem. Phys. 14, 4687 (2012).

${ }^{19}$ M. I. S. Röhr, J. Petersen, M. Wohlgemuth, V. Bonačić-Koutecký, and R. Mitrić, ChemPhysChem 14, 1377 (2013).

${ }^{20}$ J. C. Tully and R. K. Preston, J. Chem. Phys. 55, 562 (1971).

${ }^{21}$ J. C. Tully, J. Chem. Phys. 93, 1061 (1990).

${ }^{22}$ K. Drukker, J. Comput. Phys. 153, 225 (1999).

${ }^{23}$ G. Stock and M. Thoss, Adv. Chem. Phys. 131, 243 (2005).

${ }^{24}$ G. Granucci and M. Persico, J. Chem. Phys. 126, 134114 (2007).

${ }^{25}$ J. R. Schmidt, P. V. Parandekar, and J. C. Tully, J. Chem. Phys. 129, 044104 (2008).

${ }^{26}$ M. Barbatti, WIREs Comput. Mol. Sci. 1, 620 (2011)

${ }^{27}$ S. C. Cheng, C. Zhu, K. K. Liang, S. H. Lin, and D. G. Truhlar, J. Chem. Phys. 129, 024112 (2008).

${ }^{28}$ M. J. Bedard-Hearn, R. E. Larsen, and B. J. Schwartz, J. Chem. Phys. 123, 234106 (2005).
${ }^{29}$ H. M. Jaeger, S. Fischer, and O. V. Prezhdo, J. Chem. Phys. 137, 22A545 (2012).

${ }^{30}$ N. Shenvi, J. E. Subotnik, and W. Yang, J. Chem. Phys. 134, 144102 (2011).

${ }^{31}$ N. Shenvi and W. Yang, J. Chem. Phys. 137, 22A528 (2012).

${ }^{32}$ G. Granucci, M. Persico, and A. Zoccante, J. Chem. Phys. 133, 134111 (2010).

${ }^{33}$ G. Granucci, A. Toniolo, and M. Persico, J. Chem. Phys. 114, 10608 (2001).

${ }^{34}$ F. Plasser, G. Granucci, J. Pittner, M. Barbatti, M. Persico, and H. Lischka, J. Chem. Phys. 137, 22A514 (2012)

${ }^{35}$ G. Granucci, M. Persico, and G. Spighi, J. Chem. Phys. 137, 22A501 (2012).

${ }^{36}$ The value of $\Delta t$ we use in direct dynamics calculations mainly stems from the integration of the nuclear trajectory (usually $\Delta t=0.1 \mathrm{fs}$ ).

${ }^{37}$ S. Hammes-Schiffer and J. C. Tully, J. Chem. Phys. 101, 4657 (1994).

${ }^{38}$ E. J. Heller, J. Chem. Phys. 75, 2923 (1981).

${ }^{39}$ E. J. Heller, J. Chem. Phys. 62, 1544 (1975).

${ }^{40}$ W. C. Swope, H. C. Andersen, P. H. Berens, and K. R. Wilson, J. Chem. Phys. 76, 637 (1982).

${ }^{41}$ A. D. Bandrauk, E. Dehghanian, and H. Lu, Chem. Phys. Lett. 419, 346 (2006).

${ }^{42}$ M. Hillery, R. F. O'Connell, M. O. Scully, and E. P. Wigner, Phys. Rep. 106, 121 (1984).

${ }^{43}$ S. Magnier, M. Persico, and N. Rahman, Phys. Rev. Lett. 83, 2159 (1999).

${ }^{44}$ G. Granucci, S. Magnier, and M. Persico, J. Chem. Phys. 116, 1022 (2002). 